\title{
Change detection for real-world objects in perihand space
}

\author{
Stephen J. Agauas ${ }^{1} \cdot$ Laura E. Thomas ${ }^{1}$
}

Published online: 12 August 2019

(C) The Psychonomic Society, Inc. 2019

\begin{abstract}
Recent evidence has demonstrated that observers experience visual-processing biases in perihand space that may be tied to the hands' relevance for grasping actions. Our previous work suggested that when the hands are positioned to afford a power-grasp action, observers show increased temporal sensitivity that could aid with fast and forceful action, whereas when the hands are instead at the ready to perform a precision-grasp action, observers show enhanced spatial sensitivity that benefits delicate and detail-oriented actions. In the present investigation we seek to extend these previous findings by examining how object affordances may interact with hand positioning to shape visual biases in perihand space. Across three experiments, we examined how long participants took to perform a change detection task on photos of real objects, while we manipulated hand position (near/far from display), grasp posture (power/precision), and change type (orientation/identity). Participants viewed objects that afforded either a power grasp or a precision grasp, or were ungraspable. Although we were unable to uncover evidence of altered vision in perihand space in our first experiment, mirroring previous findings, in Experiments 2 and 3 our participants showed grasp-dependent biases near the hands when detecting changes to target objects that afforded a power grasp. Interestingly, ungraspable target objects were not subject to the same perihand space biases. Taken together, our results suggest that the influence of hand position on change detection performance is mediated not only by the hands' grasp posture, but also by a target object's affordances for grasping.
\end{abstract}

Keywords Perihand space $\cdot$ Grasp affordances $\cdot$ Visual search $\cdot$ Change detection $\cdot$ Visual processing

Previous work examining the processing of simple visual stimuli provides evidence that observers experience various visual/ cognitive biases in perihand space (for reviews, see Brockmole, Davoli, Abrams, \& Witt, 2013; Goodhew, Edwards, Ferber, \& Pratt, 2015; Taylor, Gozli, Chan, Huffman, \& Pratt, 2015; Tseng, Bridgeman, \& Juan, 2012). For example, early investigations provided strong evidence that observers can detect targets presented near the hands more efficiently than targets presented far from the hands (e.g., Adam, Bovend'Eerdt, van Dooren, Fischer, \& Pratt, 2012; Reed, Betz, Garza, \& Roberts, 2010; Reed, Grubb, \& Steele, 2006; Sun \& Thomas, 2013). More recent evidence has supported a theory that visual processing in perihand space is biased toward contributions from the hightemporal-acuity magnocellular (M) visual pathway, at the cost of the high-spatial-acuity parvocellular (P) pathway - the visual

Stephen J. Agauas

stephen.agauas@ndsu.edu

1 Center for Visual and Cognitive Neuroscience, Department of Psychology, North Dakota State University, Fargo, ND, USA pathways hypothesis (e.g., Abrams \& Weidler, 2013; Goodhew \& Clarke, 2016; Goodhew, Fogel, \& Pratt, 2014; Goodhew, Gozli, Ferber, \& Pratt, 2013; Gozli, West, \& Pratt, 2012). However, a series of studies from our lab suggest that the hands' positioning near stimuli can shift this visual pathways biaswhen the hands are positioned to afford a power grasp, they introduce a bias toward temporal sensitivity, whereas when they are instead positioned to afford a precision grasp, observers show increased spatial sensitivity (Thomas, 2015, 2017). Although some research has indicated that when observers prepare to execute a power or precision grasp, they show improvements in the ability to detect changes in photos of grasp-congruent objects (Symes, Tucker, Ellis, Vainio, \& Ottoboni, 2008), most studies of visual biases in perihand space have relied on very simple visual stimuli that may or may not translate to real-world applications with graspable objects. Here, we seek to extend previous findings on altered vision near the hands by testing the visual pathways hypothesis in a change detection paradigm using pictures of real, graspable objects.

The visual pathways hypothesis is supported by a growing body of literature that has attempted to examine how observers process visual information when performing tasks 
designed to rely primarily on signals carried by either the $\mathrm{M}$ or the $\mathrm{P}$ pathway, while also manipulating the observer's hand position (near vs. far from stimuli). In an initial examination of the visual pathways hypothesis, observers performed temporal and spatial gap detection tasks on a simple circular stimulus while either framing the display between the palms of their hands (i.e., near the hands) or holding their hands below the display (i.e., far from the hands; Gozli et al., 2012). As compared to the hands-far condition, observers showed increased sensitivity in detecting small temporal gaps when their hands framed the display, consistent with a bias toward the M pathway's rapid summation of signals across time intervals. However, when observers held their hands below the display, they instead showed increased sensitivity in detecting small spatial gaps, suggesting a bias toward the P pathway's high spatial acuity (Gozli et al., 2012). According to the visual pathways hypothesis, this bias toward input carried by the $\mathrm{M}$ pathway in near-hand space is adaptive: Items viewed in perihand space are candidates for immediate action, introducing a prioritization of action-relevant motion/location information (e.g., Goodhew et al., 2015). Consistent with this visual pathways hypothesis, observers show greater sensitivity to low-spatial-frequency Gabor patches in perihand space, but greater sensitivity to high-spatial-frequency patches viewed far from the hands. Interestingly, this difference in perihand space performance is essentially eliminated in the presence of red diffuse light, which suppresses activity in the M pathway (Abrams \& Weidler, 2013). Additional work supporting this hypothesis revealed that observers show increased sensitivity for orientation information on a visual working memory task performed under hands-near conditions, but increased sensitivity for color information when this task is performed under hands-far conditions (Kelly \& Brockmole, 2014).

Although these findings have advanced the visual pathways hypothesis as an explanation for processing biases in perihand space, more recent work has suggested that altered vision near the hands is also modulated by the hands' position and posture (e.g., Bush \& Vecera, 2014; Reed et al., 2010; Thomas, 2013, 2015, 2017). Most studies of visual biases in perihand space have employed a hand proximity manipulation in which observers hold their hand(s) near a display in a posture that affords a power grasp-fingers functioning as a unit that can curl around an object to secure it against the palm. This posture affords actions that are fast and forceful, actions for which information carried by the M pathway would be most relevant. However, in our lab, we have also examined visual biases associated with viewing information near hands positioned to afford a precision grasp - that is, with the thumb and forefinger at the ready to perform a more delicate and detail-oriented action that potentially relies more on information carried by the P pathway (Thomas, 2013, 2015, 2017). Consistent with previous work supporting the visual pathways hypothesis, observers show enhanced sensitivity on a global dot motion detection task but decreased sensitivity on a global dot form perception task when viewing stimuli near hands positioned in a power-grasp posture, as compared to handsfar viewing conditions. However, this pattern is reversed when participants instead hold their hands near the display in a precision-grasp posture, which introduces a bias toward spatial sensitivity at the apparent expense of temporal sensitivity (Thomas, 2015, 2017). The interaction between grasp posture and visual task suggests that grasp affordances may bias visual processing toward specific contributions from each visual pathway. Specifically, whereas power-grasp affordances may lead to a bias for increased contribution from the M pathway, precision-grasp affordances may instead introduce a bias toward contributions from the P pathway.

Although investigations of altered vision near the hands suggest that visual biases are related to the hands' relevance for action, the majority of work examining processing in perihand space has made use of simple, low-level stimuli, such as dots (e.g., Reed et al., 2006; Thomas, 2015), lines (e.g., Kelly \& Brockmole, 2014) or shapes (e.g., Gozli et al., 2012), that are not particularly grounded in real-world action. We have advanced the hypothesis that visual processing in perihand space is weighted toward contributions from the visual pathway carrying information most relevant for the current grasp affordances (Thomas, 2015). If this affordance account is correct, we should expect to see these biases reflected in the processing of displays with photos of real, graspable objects. Moreover, an examination of graspable objects enables us to investigate how grasp posture may interact with top-down knowledge about object affordances to introduce additional visual biases.

In the present investigation, we seek to extend the findings of the near-hands literature to complex stimuli using a change detection visual-search paradigm. Previous research exploring common coding between perceptual and action-based representations (e.g., Hommel, Müsseler, Aschersleben, \& Prinz, 2001) has shown that viewing objects primes performance of the actions these objects afford (e.g., Tucker \& Ellis, 2004) and that specific action planning can prime visual attention to action-relevant objects (Symes et al., 2008) or object features (Fagioli, Hommel, \& Schubotz, 2007; Wykowska, Schubö, \& Hommel, 2009). However, research within the commoncoding tradition has focused on the role that action execution or planning plays in visual processing, never examining how the hands' affordances for action-affordances arising from the hands' proximity to visual information - in the absence of an explicit requirement or plan to act can similarly affect vision. In the present study, we presented observers with photo arrays of real objects. Whereas some of these objects afforded power grasps (e.g., a soda can), others afforded precision grasps (e.g., an almond). Using a flicker paradigm, we asked participants to localize a change to either an object's orientation or its identity. According to the visual pathways 
hypothesis, performing searches under a power-grasp posture should favor the detection of orientation changes, a task primarily tapping dorsal-stream processes largely informed by the M pathway (e.g., Faillenot, Decety, \& Jeannerod, 1999), whereas precision-grasp postures should instead facilitate searches for identity changes, a task relying on ventralstream processes stemming primarily from $P$ pathway signals (e.g., Gerlach, Law, Gade, \& Paulson, 2000). In addition, if object affordances interact with the grasp affordances created by the hands' proximity to a display, then observers should be facilitated in making responses to objects that afford an action congruent with the hands' current posture-for example, responding to a soda can when the hands are positioned to afford a power grasp.

Across three experiments, we discuss the development of our change detection paradigm (Exps. 1 and 2), describe how hand proximity manipulations alter change detection performance (Exp. 2), and describe how grasp affordance manipulations affect the visual processing of object changes based on how observers interact with these objects in the real world (Exps. 2 and 3). Throughout all experiments, we recorded observers' eye movements as they searched for changes, in order to examine visual processing as it unfolded in real time. This technique allowed us to study not only the time required by observers to locate each changing object, but also how participants altered their visual search patterns on the basis of their current grasp posture and the action affordances of the objects in the array.

\section{Experiment 1: Change detection under power-grasp affordances}

Our change detection task was inspired by work from Symes et al. (2008) demonstrating that grasp planning primes visual attention to photos of grasp-congruent objects. Across a series of experiments, researchers used a flicker paradigm to examine how quickly participants detected changes when viewing photos of objects varying in size. In this investigation, observers prepared to execute a specific grasp response before viewing stimuli: Observers either performed a power-grasp response (squeezing a cylindrical button) upon identifying the changing object, or they performed a precision-grasp response (squeezing the tip of a small button). Participants were facilitated in detecting changes in objects that corresponded with the specific grasp type; that is, participants detected changes more quickly in small than in larger objects when performing a precision-grasp response (Symes et al., 2008). Using the timing and arrangement of arrays in this task as a model, we instead examined how grasp affordances - in the absence of a specific action plan - might also influence visual search behavior in a change detection flicker task.
Our first experiment presented participants with two types of target image changes: (1) orientation changes, in which the target image flipped 180 deg (e.g., a right-facing dart switched to a left-facing dart) or (2) identity changes, in which the target image was replaced by an object of similar shape and size (e.g., a pen changed into a paintbrush). The set of objects in each search array were selected to include equal numbers of items affording a power grasp (e.g., a tea kettle) and items affording a precision grasp (e.g., a pen). Participants searched for the changing object in a flickering array of images and indicated via a button press whether the target had changed its orientation or identity. Across blocks of trials, participants performed this change detection task under a hands-near condition, in which they interacted with response buttons attached to either side of the display with a power-grasp posture (see Fig. 1B), or under a hands-far condition, in which they responded using buttons resting below the display (see Fig. 1a). The visual pathways hypothesis predicts that participants should locate orientation changes more quickly than identity changes under the hands-near power affordance condition, but should locate identity changes faster than orientation changes under the hands-far condition. In addition, if object affordances interact with the hands' current grasp posture, then participants might show facilitation in detecting changes to objects in the array that require power grasps and/or show a bias toward fixating power-graspable objects under the handsnear condition.

\section{Method}

Participants The enrolled participants $(N=34)$ were North Dakota State University students from the psychology subject pool. All participants had normal vision or corrected-tonormal vision with contacts. Additionally, participants' heights were greater than five feet six inches, to allow them to comfortably reach the response buttons attached to the display from a sitting position. Upon completion of the study, participants received course credit. Two participants were removed from the data analysis due to trial losses exceeding $20 \%$, reflecting excessive inaccuracy or a lack of responses within the allotted time per trial.

Apparatus and stimuli Eye movements were recorded using an EyeLink 1000 eye tracker with a sampling rate of $1000 \mathrm{~Hz}$. Viewing was binocular, and the right eye was tracked as participants viewed each image. Stimuli were presented on a 17in. Acer computer display at $1,024 \times 768$ screen resolution controlled by an Apple Mac Mini desktop computer. Trial presentation was controlled by SR Research (2017) Experiment Builder 2.1.140. Response data were collected via button presses using a modified Gravis controller connected to the eye-tracking computer. 


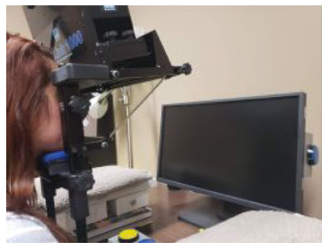

a

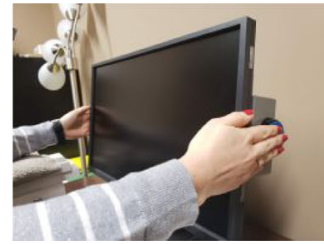

b

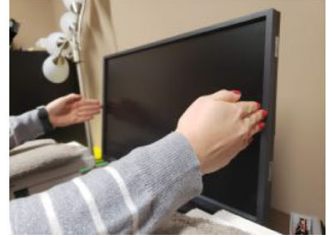

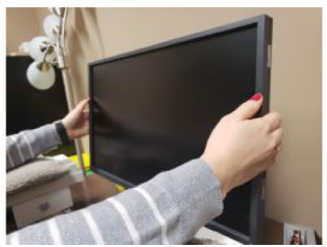

d

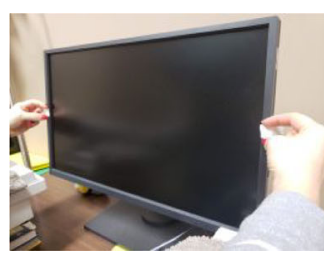

e
Fig. 1 Hand positions and affordances for Experiment 1 (a and $\mathbf{b}$ ), Experiment 2 (a, c, and $\mathbf{d})$, and Experiment 3 (a and $\mathbf{e})$. We removed buttons in Experiments 2 and 3. In Experiment 2, half of the participants

When presented with a trial, participants viewed a series of arrays containing eight approximately $150 \times 150$ pixel black-and-white photos of real objects arranged in a circle (see Fig. 2) on a white background. Within the original and modified arrays, participants viewed pictures of four power-graspable objects (e.g., a can of coke) and four precision-graspable objects (e.g., an almond). The modified array altered one of these objects via a to-be-detected orientation or identity change. Orientation changes consisted of objects rotating horizontally to face the opposite direction from the original image. Identity changes consisted of objects changing into a different object of similar shape, grasp type (i.e., power-graspable images turned into other power-graspable images), and grasp direction (i.e., if the object affords grasping the object from the side, the modified image afforded this same grasp direction). Six objects were classified as targets in the experiment: three targets that afforded a power grasp (coffee pot, hatchet, iron), and three targets that afforded a precision grasp (ring, pen, almond). To increase image diversity, we also included four different foil objects-two power- performed an open-palm power grasp position (c), and half performed a power grasp with their fingers wrapped behind the display (d). In Experiment 3, participants performed a precision grasp position (e)

graspable (can of Squirt, can of Fanta) and two precisiongraspable (dart and syringe) - that never underwent a change across the experiment.

Procedure and design Upon entering the room and providing consent, participants sat approximately $40 \mathrm{~cm}$ from the computer display. Participants rested their head on a chin rest to discourage head movement and maintain a constant viewing distance. A researcher then informed participants that on each trial they would view a series of flickering images on the computer screen, and that one of the objects in an array would change either its orientation or identity across presentations. Each trial consisted of arrays organized in a four-image cycle that included (1) an original array of objects (506 ms), (2) a blank screen $(280 \mathrm{~ms})$, (3) a modified array of objects (506 $\mathrm{ms}$ ), and (4) a final blank screen (280 ms) (see Fig. 2; the timing is identical to that in Symes et al., 2008). This cycle continued until the participant had located the changing object or until a timeout occurred after $15 \mathrm{~s}$. We instructed participants, upon locating a changing object, to fix their gaze on the object and press one of two response buttons to indicate

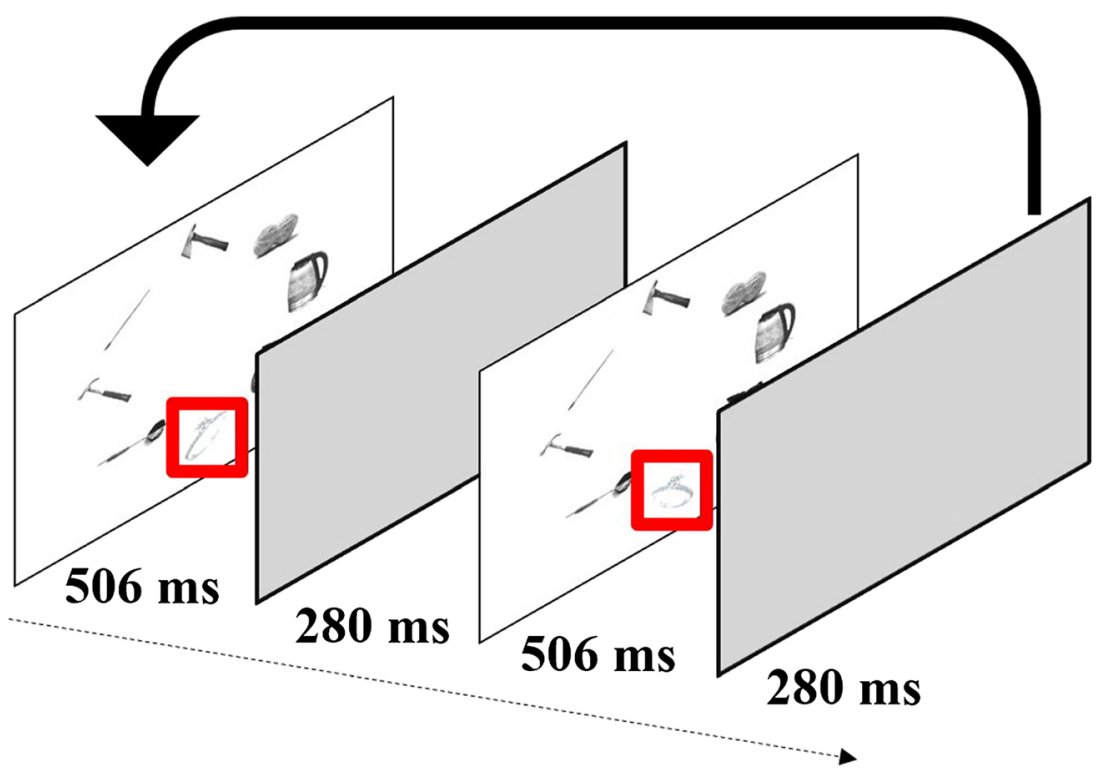

Fig. 2 Illustration of the display sequence in Experiment 1. A drift check screen appeared before the original image (not shown) 
whether the target underwent an orientation or an identity change; half of the participants responded to orientation changes with the left hand, and the other half responded to orientation changes with the right hand. To avoid any possibility of local motion cues aiding change detection, in the modified image all pictures shifted a random distance within five pixels from their original position.

All participants performed trials in which each of the possible target objects underwent an orientation change as well as an identity change. Across blocks, participants adopted either a power-grasp posture over response buttons attached to either side of the display (hands-near) or rested their hands over response buttons placed below the display (hands-far). Thus, we utilized a 2 (target type: power vs, precision) $\times 2$ (change type: orientation vs. identity) $\times 2$ (hand position: near vs. far) repeated measures design. Participants experienced each combination of target type, change type, and hand position across all eight locations for each target object.

Prior to the start of experimental trials, participants practiced the proper hand positions, covering the buttons located on the sides of the display or near them with their hands, and performed a nine-point eye-tracking calibration. A researcher guided participants through a single block of practice trials, to demonstrate the different types of changes and ensure that participants understood the requirements of the experiment, discouraging them from using the same search pattern for each image (i.e., starting at the topmost object and moving in a clockwise pattern when presented with each array). Half of the participants began the experiment with their hands far from the display, and the other half began the experiment with their hands near the display. The trials were split equally into 12 blocks (16 trials per block) in which hand position alternated between the hands-near and hands-far conditions after each block. Participants viewed a total of 16 practice trials, followed by 192 experimental trials. We performed calibrations each time participants switched their hand positions at the start of each block or if participants' gaze deviated from the drift check before each trial. The experiment lasted approximately $1 \mathrm{~h}$.

\section{Results and discussion}

Data analysis The participants' object find times and eye movement data were imported into SR Research (2018) EyeLink Data Viewer 3.1.246 and subsequently were exported into Microsoft Excel. We removed trials in which participants failed to respond within the allotted time $(10 \%$ of trials) or provided an incorrect response ( $2 \%$ of trials). We then performed a $2 \times 2 \times 2$ within-subjects analysis of variance (ANOVA) for find times, with the factors of target object type (power vs. precision), change type (orientation vs. identity), and hand position (near vs. far). For each trial, we defined eight interest areas by using elliptical sections slightly larger than each image, to avoid overlapping data. We exported the interest area reports using Data Viewer, to evaluate the percentage of total dwell time a participant spent in each nontarget interest area as well as the total percentage of fixations participants made to each interest area. The interest areas were classified on the basis of the type of grasp each array object afforded. The dwell time and number-of-fixation data did not include eye movements made viewing the target object, to avoid biasing the analysis toward time spent confirming a change to this object.

Table 1 displays the means and standard errors of the find time data for Experiment 1. The analysis of find time data revealed a main effect of type of change, $F(1,31)=75.37, p$ $<.001, \eta_{\mathrm{p}}{ }^{2}=.71$, as participants detected orientation changes $(M=3,457)$ faster than identity changes $(M=4,188)$. A main effect of target type, $F(1,31)=32.50, p<.001, \eta_{\mathrm{p}}{ }^{2}=.51$, suggested that participants detected changes more quickly in power-graspable targets $(M=3,669)$ than in precisiongraspable targets $(M=3,976)$. A main effect of hand position, $F(1,31)=6.60, p=.02, \eta_{\mathrm{p}}{ }^{2}=.18$, suggested that participants detected changes more quickly when placing their hands far from the display $(M=3,774)$ than when placing their hands near the display $(M=3,870)$. Hand position did not interact with type of change, $F(1,31)=0.54, p=.47, \eta_{\mathrm{p}}{ }^{2}=.02$, nor did it interact with target type, $F(1,31)=0.03, p=.84, \eta_{\mathrm{p}}{ }^{2}=.001$. Type of change and target type interacted significantly, $F(1$, $31)=14.52, p<.001, \eta_{\mathrm{p}}{ }^{2}=.32$; whereas participants detected power and precision orientation changes equally fast, they detected power identity changes $(M=3,931)$ more quickly than precision identity changes $(M=4,445), F(1,31)=$ $33.48, p<.001$. Additionally, when viewing power targets, participants detected orientation changes $(M=3,407)$ more quickly than identity changes $(M=3,931), F(1,31)=39.01$, $p<.001$. This orientation advantage was even more pronounced when participants viewed precision targets $(M=$ 3,506 for orientation changes, $M=4,445$ for identity changes), $F(1,31)=67.32, p<.001$. The three-way interaction between hand position, type of change, and target type was not statistically significant, $F(1,31)=0.44, p=.51, \eta_{\mathrm{p}}{ }^{2}=.01$.

Table 1 Means and standard errors for find times in Experiment 1

\begin{tabular}{lllll}
\hline Hand position & Change type & Target type & $M$ & $S E$ \\
\hline Far & Identity & Power & 3,882 & 84 \\
& & Precision & 4,429 & 112 \\
& \multirow{2}{*}{ Orientation } & Power & 3,353 & 98 \\
& & Precision & 3,433 & 99 \\
Near & Identity & Power & 3,980 & 91 \\
& & Precision & 4,461 & 111 \\
& \multirow{2}{*}{ Orientation } & Power & 3,461 & 106 \\
& & Precision & 3,579 & 111 \\
\hline
\end{tabular}

Means and $S E$ s are reported in milliseconds. $N=32$ 
Participants did not show a bias in gaze times for examining power versus precision distractors, their gaze times did not change when they placed their hands near or far from the display, and the presence of the hands did not interact with target type, $F_{\mathrm{S}}<1 .{ }^{1}$ When participants placed their hands near the display, participants spent equal proportions of their gaze time viewing power distractors $(M=50 \%)$ and precision distractors $(M=50 \%)$. The same was true when they placed their hands far from the display; participants spent equal proportions of their gaze time viewing power distractors $(M=$ $50 \%)$ and precision distractors $(M=50 \%)$. Most likely, this pattern of means is explained by the relatively few images fixated by participants during their search; that is, participants typically located the changing target after viewing one distractor object.

Although our find time data suggest that participants generally had more difficulty detecting changes in a target's identity than its orientation, and that they found changes to powergraspable targets more difficult to detect than changes to precision-graspable objects, ${ }^{2}$ we somewhat surprisingly found that neither of these factors interacted with the hands' positioning near versus far from the display. However, participants did show a general cost in response times when their hands were held near the display, perhaps as a result of experiencing greater difficulty in easily reaching/trigging the response buttons when they were in this position. In any case, the pattern of results in Experiment 1 suggests that, counter to predictions derived from the visual pathways hypothesis, the hands' grasp affordances did not selectively bias visual processing in this change detection task. This raises that possibility that, whereas the presence of the hands near simple visual stimuli introduces changes in the way in which observers process visual information, these changes may not scale up to the processing of more complex graspable objects. Although an extensive literature supports the idea that vision is biased in perihand space (e.g., Brockmole et al., 2013; Taylor et al., 2015), recent evidence has suggested that these near-hand effects may be fragile in visual search and change detection paradigms (Andringa, Boot, Roque, \& Ponnaluri, 2018) as well as in target discrimination and detection tasks (Dosso \& Kingstone, 2018), raising the possibility that small differences in procedure or a host of other potential factors may influence researchers' ability to observe visual alterations in perihand space.

We considered whether any methodological concerns with our first experiment might have introduced conditions that were not conducive to detecting near-hand effects, and noted a few potential problem sources. Although we approached the design of Experiment 1 with an eye toward a strict balancing of target

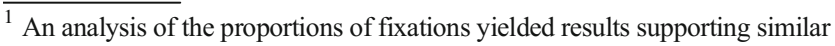
conclusions.

${ }^{2}$ It is possible these main effects could have been driven by different amounts of low-level change across the limited number of objects in each category.
}

types and changes across all locations in our arrays, this did limit the target diversity and array complexity that participants experienced, as compared to other studies investigating grasp preparation during change detection (Symes et al., 2008). In addition, posttest debriefing revealed that approximately $60 \%$ of our participants recognized that the foil objects did not change throughout the experiment and mentioned that they ignored these images after recognizing the pattern, potentially limiting/simplifying their search process. Furthermore, although investigations from other labs have successfully documented visual biases in perihand space using a setup in which participants used response buttons under the hands, as we did in Experiment 1 (e.g., Abrams, Davoli, Du, Knapp, \& Paull, 2008), previous work outside of our lab on near-hand biases had employed a near-hand power affordance condition in which participants grasped the display (Thomas, 2015, 2017). It is possible that requiring active button presses in this paradigm disrupted representations of the object grasp affordances. That is, if participants are planning a pressing action, this action could interfere with object-driven activation of grasp affordances (e.g., Tucker \& Ellis, 2004). Before concluding that the hands' posture does not influence visual processing of realworld graspable objects, we conducted a second experiment with these methodological considerations in mind.

\section{Experiment 2: Modified change detection under power-grasp affordances}

After considering the findings of Experiment 1, we adjusted our materials and apparatus to address the methodological concerns raised above, while again manipulating target type, change type, and hand position. We increased the array complexity and display size to promote more active search behavior, by including 12 items selected from a subset of 36 potential targets arranged in a configuration more similar to Symes et al.'s (2008) original design. In addition to a larger set of powerand precision-graspable targets, we also included a new class of target objects that did not afford a grasping action (e.g., live bear, lighthouse), intended to further diversify the stimulus set and also serve as a potential mechanism for evaluating the influence of graspability - in general — on the processing of objects in perihand space. We also eliminated the use of foil items that never acted as targets, to prevent participants from ruling out any objects as potential change detection targets during their searches. To investigate any potential issues with differences in power-grasp postures between Experiment 1 and our previous work (Thomas, 2015, 2017), we also introduced a new between-subjects factor of power-grasp type - half of all participants performed a hands-near condition using the openpalm posture from Experiment 1, and the remaining participants performed a hands-near condition in which they grasped the display. Finally, we did not require the participants in 
Experiment 2 to make button press responses when they located a change, an active response with the hands that might have disrupted representations of the potential object grasp affordances. Instead, once a participant had localized a change, the participant simply fixated the target for a set time to register a response and advance to the next trial.

If, as the results of Experiment 1 suggested, visual biases in perihand space do not influence change detection performance for photos of graspable objects, then we should find that our hand position manipulation did not selectively influence participants' response times to locate identity versus orientation changes. However, if issues with our first experiment's methodology prevented us from observing visual biases near the hands, or if this result fell into the category of a Type II error, we instead would expect to see that participants' hand proximity would facilitate orientation change detection more than identity change detection under hands-near conditions, but that hand proximity would facilitate identity change detection more than orientation change detection under hands-far conditions. In addition, if a target's affordances for grasping interact with the hands' current grasp posture, then participants might show an advantage for detecting changes to powergraspable targets under the hands-near condition.

\section{Method}

Participants The enrolled participants $(N=90)$ were North Dakota State University students from the psychology subject pool. ${ }^{3}$ All participants had normal vision or corrected-tonormal vision with contacts. Additionally, participants' heights were greater than five feet six inches, to allow them to reach the display from a sitting position. Upon completion of the study, participants received course credit. Ten participants were excluded from the data analysis because they did not locate the changing picture within the allotted time across more than $20 \%$ of trials. Of the remaining 80 participants, half were tested under hands-near conditions using an open palm posture similar to that in Experiment 1, in which they placed their middlemost fingers on tape indicators located on the computer display. The second half of participants performed their hands-near condition by grasping the computer display; specifically, they placed the base of their thumbs over tape indicators and wrapped their fingers as a unit around the sides of the display.

Apparatus and stimuli Eye movements were recorded using an EyeLink 1000 eye tracker with a sampling rate of $1000 \mathrm{~Hz}$. Viewing was binocular, and the right eye was tracked as

\footnotetext{
${ }^{3}$ We increased the sample size of Experiment 2 due to the reduction in observations per target image. In Experiment 1, participants had viewed each target across all eight locations. In Experiment 2, observers viewed each target image and its respective changes within one specific location on the display. To correct for this loss of power, we recruited additional participants.
}

participants viewed each image. Stimuli were presented on a Dell 23-in. LCD display at a 1,024 × 768 screen resolution, controlled by an Apple Mac Mini desktop computer. Trial presentation was controlled by the SR Research Experiment Builder.

Participants viewed a series of arrays containing 12 approximately $150 \times 150$ pixel black-and-white pictures of real objects, arranged in a $4 \times 3$ grid (see Fig. 3). Each array contained four power-graspable objects, four precisiongraspable objects, and four nongraspable objects.

Procedure and design Upon entering the room and providing consent, participants sat approximately $50 \mathrm{~cm}$ from the display. They rested their head on a chin rest to discourage head movement. A researcher then informed participants that they would be viewing a series of alternating arrays on the computer screen, and that one of the objects in the array would change its orientation or identity across alternations. As in Experiment 1, the display cycled between an initial array, a blank screen, a modified array in which a target item changed, and another blank screen. The timing of the array cycles was identical to that in the first experiment. To avoid any possibility of local motion cues aiding change detection, in the modified array all pictures shifted a random distance within ten pixels from their original position. In the modified array, the target object changed by either flipping 180 deg or by being replaced with another object of similar shape, size, and grasp affordance. The researcher instructed participants to stare at the changing image until a new trial began; we required participants to continuously view each target for 1,500 ms before recording that the participant had located the changing target. Participants then practiced the proper hand positions, covering marked areas on the right and left sides of the display with their fingers (see Fig. 1c and 1d)

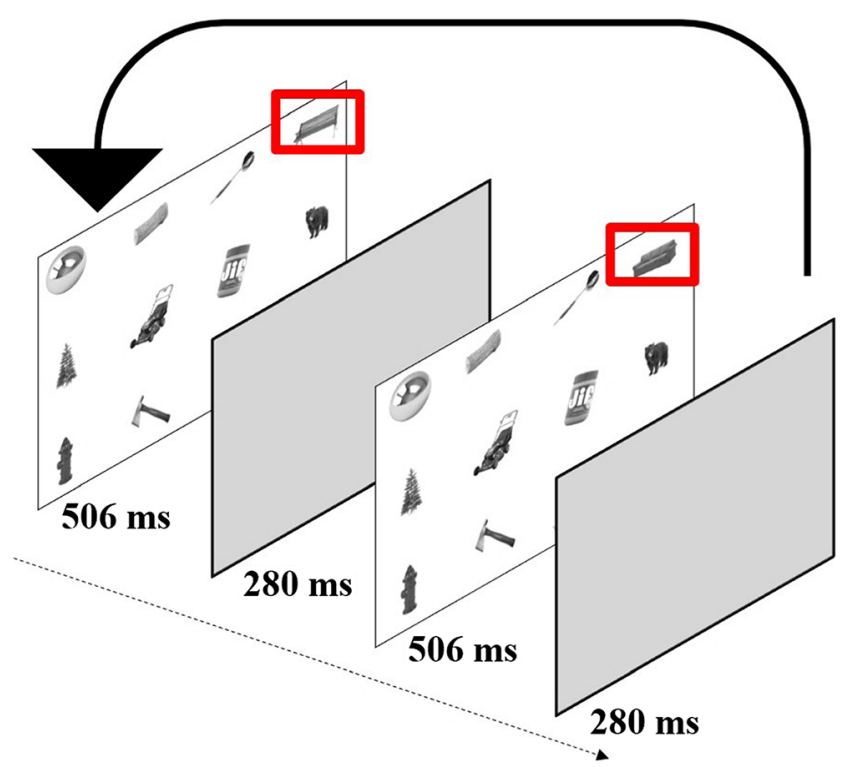

Fig. 3 Illustration of the display sequence in Experiments 2 and 3. A drift check screen appeared before the original image (not shown) 
and performed a nine-point calibration procedure. Before each trial, participants viewed a drift correct screen to confirm that their eye position had not changed.

All participants viewed 36 total target objects (12 each of power-graspable, precision-graspable, and nongraspable). Each target object was presented once as an identity change and once as an orientation change when participants placed their hands near the display; the target objects were also presented as identity/orientation changes when participants placed their hands far from the display. Thus, we utilized a 3 (target type) $\times 2$ (change type) $\times 2$ (hand position) repeated measures design. Participants viewed all conditions once in each of the 12 locations of the $4 \times 3$ array, for a total of 12 observations per condition. The trials were split equally into 12 blocks ( 12 trials per block), and participants alternated their hand position after each block. We repeated the calibration procedure each time participants shifted their hand position before the start of each block, or if participants' gaze deviated from the drift check before each trial. Half of our participants began the experiment with their hands near the display in either the open-palm or display-grasping power posture, and the other half began the experiment with their hands far from the display. Participants viewed a total of six guided practice trials, followed by 144 experimental trials in total. The experiment lasted approximately $1 \mathrm{~h}$.

\section{Results and discussion}

Data analysis The participant eye movement data were imported into SR Research Data Viewer 3.1.246 and subsequently exported data into Microsoft Excel. Trials were excluded from the data analysis if participants activated the fixation trigger before completing a full cycle of arrays including boundary fixation time $(<2,500 \mathrm{~ms} ; \sim 3 \%$ of the data) or if participants did not locate the changing object within $15 \mathrm{~s}$ (10\% of the data). Trials in which the targets appeared to the immediate left or right of the central drift correction location were excluded from the analyses due to participants' frequent failure to visit any distractor items during these trials. We defined the find time for each trial by subtracting the time marked when participants viewed the original image from the time marked when participants located the changing target. After averaging the find time measure across all change locations, we performed a $3 \times 2 \times 2 \times 2$ mixed ANOVA on the data. Target type (power vs. precision vs. ungraspable), change type (orientation vs. identity), and hand position (near vs. far) were included as within-subjects factors, and powergrasp type (open-palm vs. display grasp) was included as a between-subjects factor.

Table 2 displays the means and standard errors of find time data for Experiment 2. Although we included power-grasp type as a between-subjects factor, we found no main effect of this factor, nor did it interact with any of the within- subjects variables across the ANOVA, all $p$ s $<.21$ (see Appendix 2 for inferential statistics with grasp type in the model). We therefore report means and inferential statistics for a $2 \times 2 \times 3$ repeated measures ANOVA after removing this between-subjects factor from the model. Mirroring findings from Experiment 1, our analysis revealed a main effect of change type, $F(1,79)=7.56, p=.01, \eta_{\mathrm{p}}{ }^{2}=.09$, in which participants detected orientation changes $(M=6,263)$ faster than identity changes $(M=6,427)$, and a main effect of target type, $F(2,158)=21.60, p<.001, \eta_{\mathrm{p}}{ }^{2}=.22$, that suggested that participants detected changes more quickly for precisiongraspable items $(M=6,118)$ than for power-graspable items $(M=6,364)$ and ungraspable items $(M=6,553)$. Post-hoc planned comparisons confirmed that these differences were statistically significant, as participants detected changes more quickly for precision than for power objects, $t(79)=3.68$, Bonferroni-corrected $p=.001$, Cohen's $d_{z}=0.41$, and more quickly than ungraspable objects, $t(79)=5.97$, Bonferronicorrected $p<.001$, Cohen's $d_{z}=0.67$. Additionally, participants detected changes more quickly to power than to ungraspable objects, $t(79)=3.23$, Bonferroni-corrected $p=.01$, Cohen's $d_{z}=0.36$. A main effect of hand position failed to reach significance, $F(1,79)=0.04, p=.84, \eta_{\mathrm{p}}{ }^{2}=.001$.

Departing from our findings in Experiment 1, in Experiment 2 we found that hand position interacted significantly with change type, $F(1,79)=6.30, p=.01, \eta_{\mathrm{p}}{ }^{2}=.07$ (see Fig. 4). To explore this interaction, we performed a series of post-hoc simple main effect tests. The first ANOVA examined the effect of change type when participants placed their hands near the display, and the second examined the effect of change type when participants placed their hands far from the display. These tests provided evidence that this interaction was driven by an advantage in detecting orientation changes

Table 2 Means and standard errors for find times in Experiment 2

\begin{tabular}{lllll}
\hline Hands & Change type & Target type & $M$ & $S E$ \\
\hline Far & Identity & Power & 6,365 & 107 \\
& & Precision & 6,408 & 102 \\
& & Ungraspable & 6,255 & 88 \\
& \multirow{4}{*}{ Orientation } & Power & 6,163 & 111 \\
& & Precision & 6,075 & 123 \\
& & Ungraspable & 6,760 & 107 \\
Near & Identity & Power & 6,765 & 117 \\
& & Precision & 6,202 & 110 \\
& & Ungraspable & 6,564 & 119 \\
& \multirow{3}{*}{ Orientation } & Power & 6,161 & 106 \\
& & Precision & 5,785 & 111 \\
& & Ungraspable & 6,631 & 123 \\
& & &
\end{tabular}

Means are collapsed across power grasp types (traditional vs. wrap) because grasp type failed to interact significantly with other IVs. Means and $S E$ s are reported in milliseconds. $N=80$ 


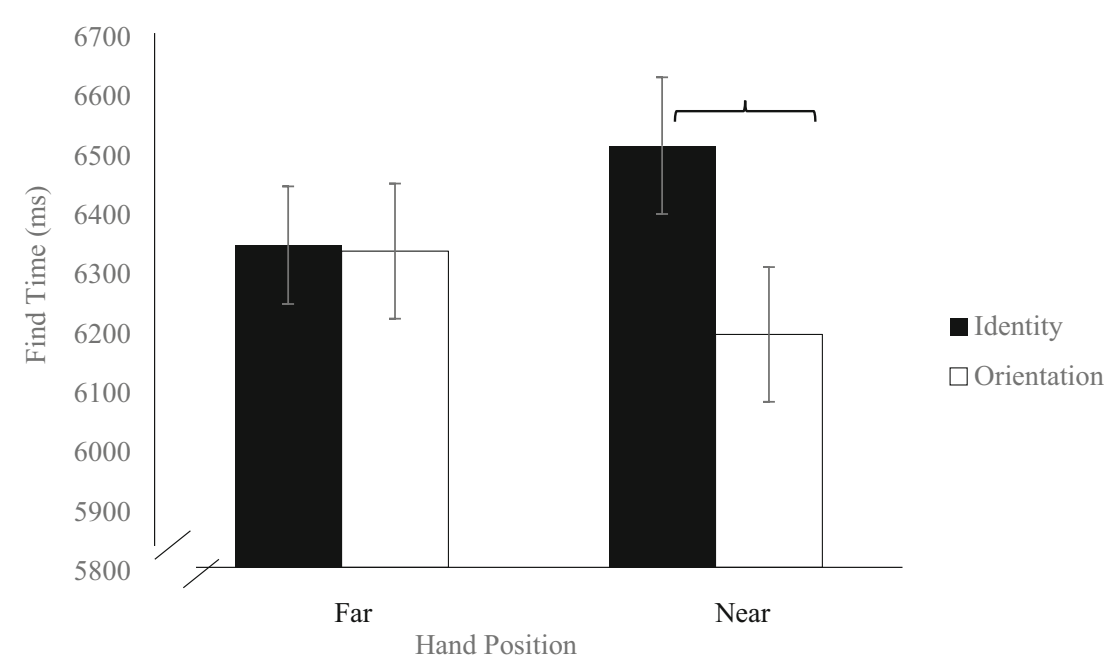

Fig. 4 Means and SEs for the interaction effect between hand position and change type in Experiment 2. Statistically significant pairwise comparisons are marked, $p<.05$. Error bars represent standard errors of the means

under the hands-near condition, $F(1,79)=16.77, p<.001(M$ $=6,192$ for orientation changes, $M=6,510$ for identity changes). Under the hands-far condition, participants were no faster to detect orientation than identity changes, $F(1,79)=0.01, p=$ .91 . These results provide partial support of the visual pathways hypothesis, suggesting that viewing objects near hands positioned to afford a power grasp introduced a bias toward information carried by the $\mathrm{M}$ pathway that benefited the localization of orientation changes.

Interestingly, participants' hand position also interacted significantly with target type, $F(2,158)=6.65, p=.002, \eta_{\mathrm{p}}{ }^{2}$ $=.08$ (see Fig. 5). Under the hands-far condition, we observed an effect of target type, $F(2,158)=5.67, p=.004$. Whereas participants detected changes in power and precision targets in similar times, they took longer to identify ungraspable object changes $(M=6,507)$ than to identify changes to either powergraspable targets $(M=6,264), t(79)=2.82$, Bonferronicorrected $p=.02$, Cohen's $d_{z}=0.32$, or precision-graspable targets $(M=6,242), t(79)=3.22$, Bonferroni-corrected $p=$ .01 , Cohen's $d_{z}=0.36$. However, when participants placed their hands near the display in a power-grasp posture, we observed a stronger effect of target type, $F(2,158)=21.57$, $p<.001$. Participants detected changes more quickly to precision targets $(M=5,994)$ than to either power targets $(M=$ $6,463), t(79)=4.87$, Bonferroni-corrected $p<.001$, Cohen's $d_{z}=0.55$, or ungraspable targets $(M=6,598), t(79)=5.78$, Bonferroni-corrected $p<.001$, Cohen's $d_{z}=0.64$. To be clear, participants took marginally longer to detect changes when viewing power targets near versus far from the hands, $F(1$, $158)=3.52, p=.06$, but participants significantly improved their time to locate changes when viewing precision targets near versus far from the hands, $F(1,158)=6.53, p=.01$.

In addition to these interactions with hand position, we also found a significant interaction between change type and target type, $F(2,158)=14.23, p<.001, \eta_{\mathrm{p}}^{2}=.15$. This interaction was driven primarily by ungraspable targets: Participants detected power object orientation changes more quickly than power object identity changes, $F(1,158)=13.80, p<.001$, and the same was true of the precision objects, $F(1,158)=13.86, p<.001$. However, participants spent more time locating ungraspable targets when the objects underwent an orientation rather than an identity change, $F(1,158)=8.07, p=.01$. This pattern is likely explained by the fact that our set of ungraspable targets contained a large number of fairly symmetrical items that yielded relatively more subtle orientation changes.

The three-way interaction between hand position, type of change, and image type failed to reach statistical significance, $F(2,158)=1.19, p=.31, \eta_{\mathrm{p}}^{2}=.02$.

As in Experiment 1, we examined the times that participants spent looking at the various distractor objects in our displays via an analysis of dwell time proportions and fixation percentages. For the proportion-of-dwell-time dependent variable, we found a main effect of distractor object type, $F(2,158)=52.72, p<$ $.001, \eta_{\mathrm{p}}{ }^{2}=.40$. Overall, participants spent an increased proportion of their dwell time examining ungraspable distractors $(M=$ $35 \%)$, as compared to both power-graspable distractors $(M=$ $33 \%), t(79)=6.74, p<.001$, Cohen's $d_{z}=0.75$, and precisiongraspable distractors $(M=31 \%), t(79)=9.06, p<.001$, Cohen's $d_{z}=1.01{ }^{4}$ Additionally, participants spent an increased proportion of their dwell time examining power-graspable distractors $(M=33 \%)$, as compared to precision-graspable distractors $(M=$ $31 \%), t(79)=3.81, p<.001$, Cohen's $d_{z}=0.43$. Hand position did not affect find times overall, nor did it interact with image type when examining proportions of dwell time, $F<0.75$. When we examined fixation proportions, participants had a slightly higher proportion of fixations on all distractors when placing their hands far from the display $(M=33.4 \%)$, relative to

\footnotetext{
${ }^{4}$ Participants may have spent more time fixating nongraspable distractor objects because of a relative difficulty of finding orientation changes to these items, or because these objects were more visually complex.
} 


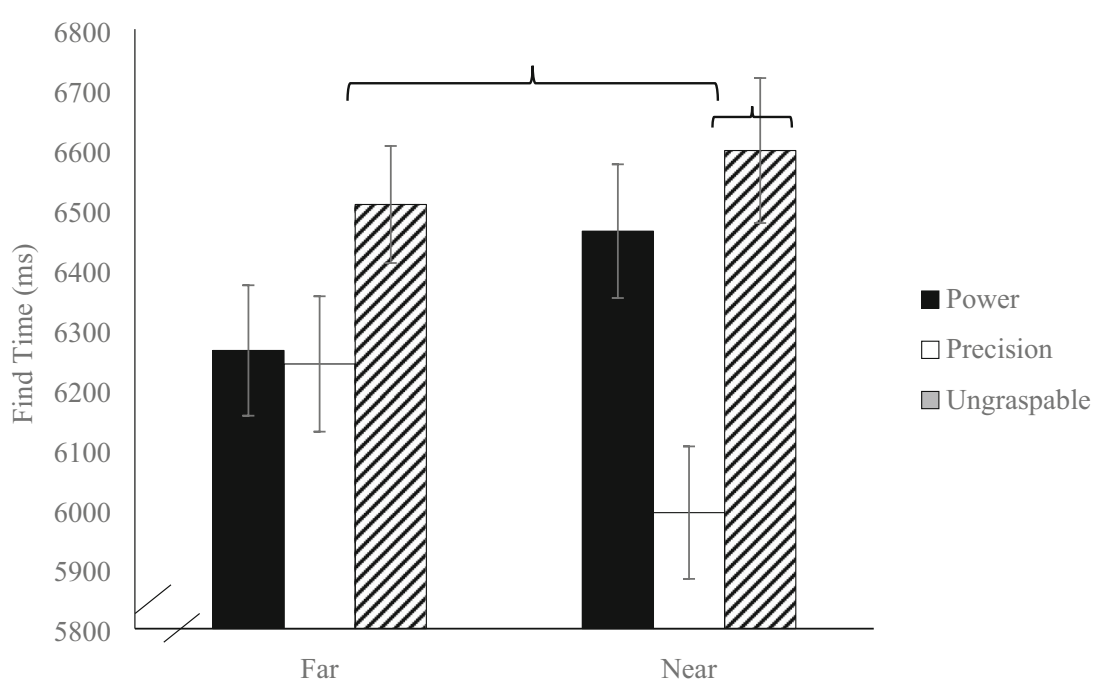

Fig. 5 Means and $S E$ s for the interaction effect between hand position and target type in Experiment 2. Statistically significant pairwise comparisons are marked, $p<.05$. Error bars represent standard errors of the means

when they placed their hands near the display $(M=33.3 \%)$, $F(1,79)=4.38, p=.05, \eta_{\mathrm{p}}^{2}=.01$. We also found a main effect of distractor object type, $F(2,158)=29.30, p<.001, \eta_{\mathrm{p}}{ }^{2}=.27$. Participants spent an increased proportion of their fixations examining ungraspable distractors $(M=35 \%)$ as compared to both power-graspable distractors $(M=33 \%), t(79)=4.06, p<$ .001 , Cohen's $d_{z}=0.45$, and precision-graspable distractors ( $M$ $=32 \%), t(79)=6.71, p<.001$, Cohen's $d_{z}=0.75$. Additionally, participants spent an increased proportion of their fixations examining power-graspable as compared to precision-graspable distractors, $t(79)=4.29, p<.001$, Cohen's $d_{z}=0.48$. The position of the hands and distractor object type did not interact, $F(2,158)=1.99, p=.14, \eta_{\mathrm{p}}{ }^{2}=.03$.

Countering the null findings concerning hand position in Experiment 1, the participants in our second experiment showed evidence of visual biases in perihand space, suggesting that our changes in methodology may have been effective in creating conditions more conducive to yielding near-hand effects. The significant interaction between hand position and change type in the present experiment is consistent with predictions stemming from the visual pathways hypothesis, in that participants located orientation changes more quickly than identity changes when they placed their hands near the display. Interestingly, the interaction we observed between hand position and target type was not consistent with our predictions regarding facilitation in detecting changes with grasp-affordance-congruent targets (i.e., a power target advantage in hands-near conditions). However, an examination of the find times for different types of target objects intriguingly suggests that an object's affordances $d o$ impact processing. To further explore these interactions, provide an opportunity to replicate the finding of altered vision in perihand space with our updated methodology, and test our predictions regarding the impacts of different grasp affordances on near-hand effects, we conducted a final experiment in which participants performed the change detection task using a precisiongrasping posture.

\section{Experiment 3: Modified change detection under precision-grasp affordances}

Recent work from our lab (Thomas, 2015, 2017) suggested that visual biases in perihand space are shaped by the hands' affordances for different grasping actions. That is, viewing information near hands positioned to afford a power grasp introduces biases consistent with increased contributions from the high-temporal-sensitivity M pathway, whereas viewing information near hands at the ready to perform a precision grasp instead biases processing toward increased contributions from the high-spatial-acuity $\mathrm{P}$ pathway. The results of Experiment 2 support the idea that observers more readily detect changes in an object's orientation - changes relying on M pathway/dorsal-stream processes - when these changes occur within the hands' power-grasping space. In Experiment 3 , we explored whether this apparent bias in change detection would shift when participants instead viewed objects near hands positioned to afford a precision grasp. If, as our previous research suggested, precision-grasp affordances introduce biases toward $\mathrm{P}$ pathway/ventral-stream processing, then we should see that participants showed a relative advantage in localizing identity changes when these changes were viewed near hands positioned to afford a precision grasp. In Experiment 2, we also observed the somewhat counterintuitive finding that holding the hands near the display in a powergrasp posture facilitated participants' ability to localize changes to targets that afforded a precision grasp. In Experiment 3, we examined whether viewing stimuli under precision-grasp 
posture conditions would introduce a similar bias, or would perhaps instead induce a bias toward detecting changes to power-graspable targets.

\section{Method}

Participants The enrolled participants $(N=36)$ were North Dakota State University students from the psychology subject pool. A power analysis (G-Power) calculated using the effect size of the critical Hands $\times$ Change Type interaction of Experiment $2(f=0.28)$ indicated that a sample of 30 participants would yield .95 power at $\alpha=.05$. All participants had normal vision or corrected-to-normal vision with contacts. Additionally, participants' heights were greater than five feet six inches to allow them to reach the display from a sitting position. Upon completion of the study, participants received course credit. Six participants were excluded from the data analysis because they did not locate the changing picture within the allotted time across more than $20 \%$ of trials, leaving us with a final sample size of 30 .

Apparatus, stimuli, procedure, and design Experiment 3 was a direct replication of Experiment 2 with one key difference: Whereas the participants in the second experiment had adopted a hands-near posture in which they were at the ready to perform a power grasp, the hands-near condition of Experiment 3 entailed participants holding their forefinger and thumb over tape located on both sides of the computer display in a precision-grasping posture (see Fig. 1e).

\section{Results and discussion}

Data cleaning and analysis The data-cleaning and analysis procedures were identical to those in Experiment 2. Trials were excluded from the data analysis if participants activated the fixation trigger before viewing a full cycle of arrays, including boundary fixation time $(<2,500 \mathrm{~ms} ; \sim 5 \%$ of the data), or if participants did not locate the changing object within $15 \mathrm{~s}$ (10\% of the data).

Table 3 displays the means and standard errors of the find time data for Experiment 3. A 3 (target type: power vs. precision vs. ungraspable) $\times 2$ (change type: orientation vs. identity) $\times 2$ (hand position: hands-near-precision vs. hands-far) repeated measures ANOVA on these data failed to detect main effects of change type, $F(1,29)=0.13, p=.72, \eta_{\mathrm{p}}{ }^{2}=.01$; target type, $F(2$, $58)=1.28, p=.29, \eta_{\mathrm{p}}{ }^{2}=.04$; or hand position, $F(1,29)=0.97$, $p=.33, \eta_{\mathrm{p}}{ }^{2}=.03$. Additionally, the presence of the hands did not interact with change type or target type, $F_{\mathrm{S}}<0.3$. Change type interacted marginally with target type, $F(2,58)=2.56, p=$ $.09, \eta_{\mathrm{p}}{ }^{2}=.04$, although an examination of Table 3 suggests that this was due to participants' difficulty in detecting ungraspable orientation changes, when compared to the power and precision images. However, we did observe a significant three-way
Table 3 Means and standard errors for find times in Experiment 3

\begin{tabular}{lllll}
\hline Hands & Change type & Target type & $M$ & $S E$ \\
\hline Far & Identity & Power & 7,072 & 190 \\
& & Precision & 6,550 & 170 \\
& & Ungrasp & 6,669 & 167 \\
& \multirow{3}{*}{ Orientation } & Power & 6,411 & 149 \\
& & Precision & 6,682 & 217 \\
& & Ungrasp & 6,936 & 216 \\
\multirow{3}{*}{ Near } & Identity & Power & 6,437 & 174 \\
& & Precision & 6,848 & 187 \\
& & Ungrasp & 6,646 & 180 \\
& \multirow{3}{*}{ Orientation } & Power & 6,685 & 241 \\
& & Precision & 6,335 & 213 \\
& & Ungrasp & 6,937 & 219 \\
\hline
\end{tabular}

Means and SEs are reported in milliseconds. $N=30$

interaction between hand position, change type, and target type, $F(2,58)=4.83, p=.01, \eta_{\mathrm{p}}{ }^{2}=.14$. Interestingly, the influence of hand position on detecting the different change types differed when these changes occurred in power versus precision versus ungraspable targets.

Breaking down this three-way interaction (see Fig. 6b, $\mathrm{d}, \mathrm{f}$ ), we found that when participants viewed targets that afforded a power grasp, identity changes $(M=7,072)$ took longer to detect than orientation changes $(M=6,411)$ if participants placed their hands far from the display, $F(1$, $29)=10.36, p=.003$. However, when participants placed their hands near the display in the precision-grasp posture, the times required to detect identity $(M=6,437)$ and orientation $(M=6,685)$ changes were similar, $F(1,29)=1.29, p$ $=.27$. Thus, participants located power targets that changed identity significantly faster when they placed their hands near the display than when they placed their hands far from the display, $F(1,29)=10.57, p=.003$.

The influence of hand position on find times for orientation versus identity changes was markedly different when the changes instead occurred on targets that afforded a precision grasp. Primarily, we failed to detect a significant interaction between hand position and change type when observers viewed precision targets, $F(1,29)=2.87, p=.10, \eta_{\mathrm{p}}{ }^{2}=.09$. When participants placed their hands far from the display, their find times were similar for identity $(M=6,550)$ versus orientation $(M=6,682)$ changes, $F(1,29)=0.29, p=.59$. However, when participants placed their hands near the display in the precision-grasp posture, they detected orientation changes $(M=6,335)$ more quickly than identity changes $(M=$ $6,848)$, although this trend failed to reach statistical significance, $F(1,29)=2.64, p=.12$. Participants showed a marginally significant trend in improving their time to locate orientation changes when they placed their hands near versus far from the display, $F(1,29)=3.11, p=.09$. 
Power Grasp - Power Target

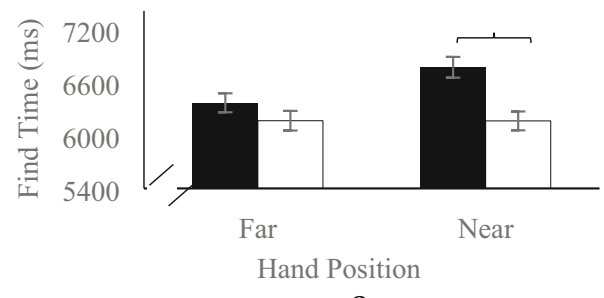

a

Power Grasp - Precision Target

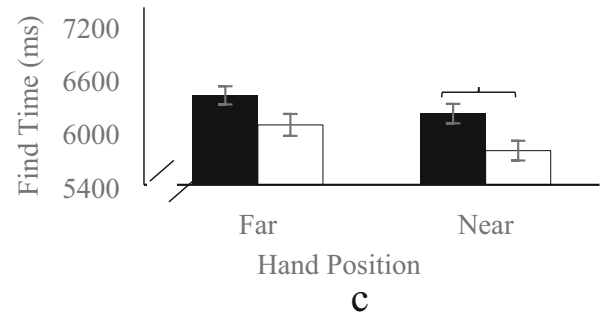

Power Grasp - Ungraspable Target

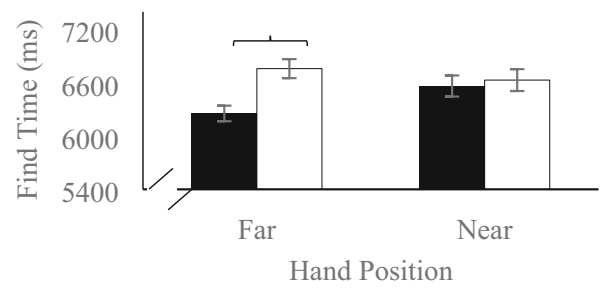

e

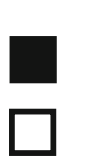

Fig. 6 Means and $S E$ s for the Hand Position $\times$ Change Type $\times$ Target Type $\times$ Grasp Type interaction. The left column $(\mathbf{a}, \mathbf{c}, \mathbf{e})$ represents the data from Experiment 2 (power grip), and the right column (b, d, f)

When examining the influence of hand position on find times for changes to ungraspable targets, we found essentially no evidence that these targets were processed differently when the hands were placed near versus far from the display, $F(1$, 29) $=0.006, p=.94, \eta_{\mathrm{p}}{ }^{2}=.00$.

An examination of participants' patterns of looking behavior on distractor items mirrored the findings of Experiment 2. Participants did not change their looking behavior when placing their hands near or far from the display overall; this was the case for both dwell times (near $M=33 \%$, far $M=33 \%$ ), $F(1,29)=0.08, p=.78, \eta_{\mathrm{p}}^{2}<.01$, and fixation proportions $($ near $M=33 \%$, far $M=33 \%), F(1,29)=2.78, p=.11, \eta_{\mathrm{p}}{ }^{2}=$ .09. As in the previous experiment, we found a main effect of distractor type on the proportions of dwell time, $F(2,58)=$ $29.48, p<.001, \eta_{\mathrm{p}}{ }^{2}=.50$. Overall, participants spent an increased proportion of their dwell time examining ungraspable distractor objects $(M=36 \%)$, as compared to both powergraspable objects $(M=33 \%), t(29)=6.03, p<.001$,
Precision Grasp - Power Target

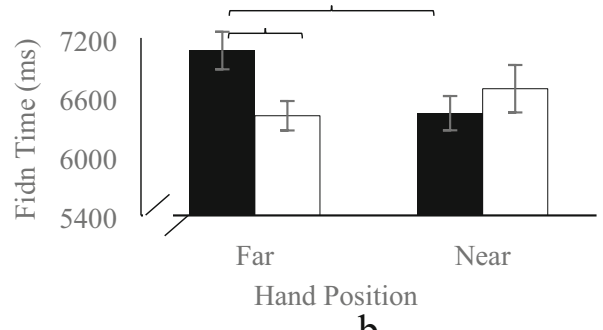

b

Precision Grasp - Precision Target

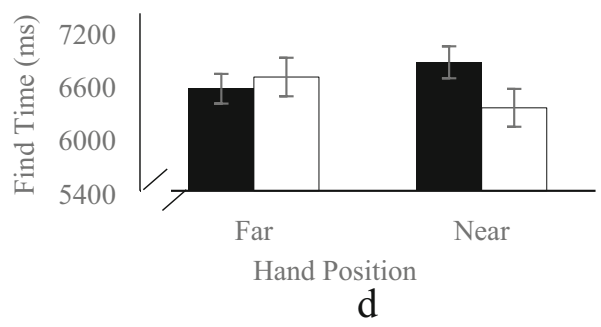

Precicion Grasp - Ungraspable Target

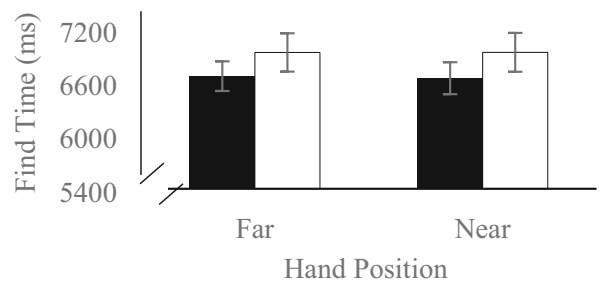

Change Type

f

Identity

Orientation

represents the data from Experiment 3 (precision grip). The rows represent target types: top row $=$ power targets, middle row $=$ precision targets, bottom row $=$ ungraspable targets

Cohen's $d_{z}=1.01$, and precision-graspable objects $(M=$ $31 \%), t(29)=6.65, p<.001$, Cohen's $d_{z}=1.21$. Additionally, participants spent an increased proportion of their dwell time examining power-graspable as compared to precision-graspable objects, $t(29)=2.98, p=.02$, Cohen's $d_{z}=$ 0.55. As in Experiment 2, the hands' position did not interact with image type when examining proportions of dwell time, $F(2,58)=0.25, p=.78, \eta_{\mathrm{p}}{ }^{2}=.01$, or when examining fixation proportions, $F(2,58)=0.54, p=.58, \eta_{\mathrm{p}}^{2}=.02$. When examining proportions of fixations, we also found a main effect of distractor type, $F(2,58)=8.86, p<.001, \eta_{\mathrm{p}}{ }^{2}=.23$. Overall, participants spent an increased proportion of their fixations examining ungraspable distractor objects $(M=35 \%)$, as compared to both power-graspable objects $(M=33 \%), t(29)=$ $3.51, p=.005$, Cohen's $d_{z}=0.64$, and precision-graspable objects $(M=32 \%), t(29)=3.55, p=.004$, Cohen's $d_{z}=$ 0.65 . However, participants spent similar proportions of fixations examining power and precision targets, $t(29)=1.56, p=$ 
39, Cohen's $d_{z}=0.28$. The hands' position did not interact with image type when examining proportions of dwell time, $F(2,58)=0.25, p=.78, \eta_{\mathrm{p}}{ }^{2}=.01$, or when examining fixation proportions, $F(2,58)=0.54, p=.58, \eta_{\mathrm{p}}{ }^{2}=.02$.

The findings of Experiment 3 provide additional evidence that visual biases in perihand space can occur when observers view stimuli more complex than simple lines or dots. Importantly, these findings also suggest that the hands' affordances for grasping shape the visual biases we observe near the hands, and may also interact with the action affordances of objects in perihand space. Specifically, whereas we observed an interaction between our hand position manipulation and change type in the predicted direction when participants detected changes in targets affording a power grasp, the influence of the hands' position was marginal or nonexistent for precision-graspable and ungraspable targets, respectively. In other words, participants only showed a bias consistent with our predictions about facilitated $\mathrm{P}$ pathway/ventral processing for targets viewed near hands in the precision posture when these targets afforded a power grasp: Participants were facilitated in detecting identity changes near hands adopting a precision-grasp posture in this condition. This result contrasts with our findings in Experiment 2, in which the power-grasp posture generally facilitated the detection of targets undergoing orientation changes. However, before we can conclude that the visual biases associated with detecting changes near hands positioned to afford a power grasp are truly different from the visual biases associated with viewing images changing near hands in a precision-grasp posture, we must directly compare the findings of Experiments 2 and 3 in an omnibus analysis.

To this end, we combined the find time data for the second and third experiments and performed an additional 3 (target type: power vs. precision versus ungraspable) $\times 2$ (change type: orientation vs. identity) $\times 2$ (hand position: hands-near vs. hands-far) $\times 2$ (grasp affordance: power vs. precision) mixed ANOVA using grasp affordance as a between-subjects factor. The four-way interaction between hand position, type of change, target image grasp type, and grasp affordance was statistically significant, $F(2,216)=6.65, p=.002, \eta_{\mathrm{p}}{ }^{2}=.06$ (see Fig. 6). Crucially, this interaction indicates that the influence of the hands' positioning on change detection performance in these tasks depends on whether the hands are positioned to afford a power or a precision grasp. That is, the near-hands effect in Experiment 2 (power-grasp posture) is itself significantly different from the near-hands effect in Experiment 3 (precision-grasp posture). To further explore this significant four-way interaction, we ran three separate $2 \times 2 \times 2$ mixed ANOVAs, with the within-subjects factors of change type (orientation vs. identity) and hand position (near vs. far) and a between-subjects factor of grasp affordance (power vs. precision), for each of our target object types. We were potentially interested in 24 pairwise comparisons available in this followup analysis (i.e., identity vs. orientation changes under each hand posture, as well as down vs. up for each change type) and made a conservative choice to use a Bonferroni correction and determined a critical $p$ value of .002 . We discuss only the pairwise comparisons approaching or exceeding statistical significance based on this Bonferroni correction.

When examining power targets, we detected an interaction between hand position, change type, and grasp type, $F(1,108)$ $=13.69, p<.001, \eta_{\mathrm{p}}{ }^{2}=.11$ (Fig. 6a and b). The interaction between hand position and change type differed when participants performed a power versus a precision grasp. Participants who adopted a power-grasp posture in the hands-near condition detected orientation changes $(M=$ $6,161)$ faster than identity changes $(M=6,765), F(1,108)=$ 20.02, $p<.001$. However, participants who adopted a precision-grasp posture in the hands-near condition were marginally faster to detect identity changes when their hands were near $(M=6,437)$ rather than far from $(M=7,072)$ the display, $F(1,108)=10.36, p=.003$.

When examining precision (Fig. 6c and d) and ungraspable (Fig. 6e and f) targets, we found no evidence for three-way interactions between hand position, change type, and grasp type $[F(1,108)=2.04, p=.16$, for precision targets; $F(1$, $108)=1.52, p=.22$, for ungraspable targets]. However, planned Bonferroni-corrected pairwise comparisons indicated that participants showed facilitation in detecting orientation $(M=5,785)$ versus identity $(M=6,202)$ changes under the power-grasp hands-near condition, $F(1,108)=9.83, p=.002$, that mirrored the facilitation present for power targets. In addition, the participants in Experiment 2 were faster to detect ungraspable objects undergoing an identity change $(M=$ $6,255)$ than an orientation change $(M=6,760)$ in the handsfar condition, $F(1,108)=13.94, p<.001$ (Fig. 6e).

\section{General discussion}

The literature on altered vision near the hands argues that observers experience biases when viewing stimuli within versus outside the hands' grasping space, due to an adaptive sensitivity to behavioral contexts: Visual information that is most relevant to an observer's current affordances for action receives enhanced processing (e.g., Thomas, 2015). Although research on visual near-hand effects appeals to action-based explanations presumably grounded in interactions with objects, investigations have typically relied on simple stimuli without clear real-world counterparts (e.g., Abrams et al., 2008; Gozli et al., 2012; Reed et al., 2006). Across three experiments, we investigated visual biases in perihand space when observers viewed photos of real objects that do or do not afford grasping actions. Although our initial experiment failed to provide evidence of a near-hand effect, across two additional experiments, participants' ability to detect changes to an object's orientation or identity depended not only on whether 
the object was presented near or far from their hands, but also on whether the hands were at the ready to perform a power or precision grasp. Moreover, these near-hand effects were mediated by the grasping action that the object afforded.

On the basis of previous findings, we hypothesized that hand postures that afford different types of actions would differentially bias vision (Thomas, 2015), with power-grasp postures facilitating orientation change detection and precision-grasp postures facilitating identity change detection. The data from observers detecting changes to targets that afford a power-grasping action support this hypothesis: Participants detected orientation changes more quickly than identity changes when placing their hands near the display in a power-grasp posture, but detected identity changes more quickly when they placed their hands near the display in a precision-grasp posture than when their hands were far from the display. This pattern is consistent with the idea that viewing objects near hands positioned to afford a power grasp biases visual processing toward an increased contribution from $\mathrm{M}$ pathway/dorsal processes, whereas precision-grasp postures instead bias visual processing toward P pathway/ventral processes (e.g., Gozli et al., 2012; Thomas, 2015).

Interestingly, participants' ability to detect changes to objects not readily affording a simple grasping action was relatively unaffected by the hands' proximity to the display. When participants held their hands near the display in a power-grasp posture, find times were similar for orientation and identity changes to ungraspable targets. Likewise, there was no significant difference in the time it took participants to detect orientation versus identity changes to ungraspable targets when they held their hands near the display in a precision-grasp posture. Taken together with the pattern of data from powergraspable targets, these intriguing null effects suggest that, at least for the case of real-object photos, top-down information about object affordances may mediate near-hand visual biases. That is, whereas the visual system apparently biased processing of objects that afford a power-grasping action on the basis of the hands' current action capabilities, objects without a clear grasp affordance were not subject to such biases.

Although the results for targets that afford a power grasp and ungraspable targets point to intriguing interactions between near-hand visual biases and viewed-object affordances, the pattern of data for precision target objects was less consistent with our predictions. When participants viewed changes to these targets while holding their hands near the display in a power-grasp posture, they were facilitated in detecting orientation relative to identity changes. However, no such biases were apparent when participants instead held their hands near the display in a precision-grasp posture. Why might this be the case? Although we can only speculate on the basis of our data, one possibility is that the grasp affordances of our precision targets were less apparent than the affordances of our power targets. Whereas a majority of the power targets that participants viewed had handles that presumably activated grasping motor representations (e.g., Tucker \& Ellis, 1998), the precision targets' graspability may have been less salient or robust; our present data do not directly address the extent to which individual targets invoked the affordances we ascribed to them. In addition, our photos were all sized to occupy roughly the same area on the display, yet they represented objects with vastly different real-world sizes, potentially weakening the representations of affordances. In future work, we hope to investigate how the presented size of an object may interact with near-hand effects.

Future work will also be necessary to establish the boundary conditions for observing altered vision near the hands. Although the results of Experiments 2 and 3 did provide evidence that the presence of the hands influenced participants' relative speed at detecting orientation versus identity changes, we found no such interaction between hand position and change type in our first experiment. Coupled with other, recent failures to find near-hand effects in the literature (Andringa et al., 2018; Dosso \& Kingstone, 2018), this result suggests that perihand visual biases may not be robust under all conditions. We implemented several design changes between Experiment 1 and Experiment 2, including increasing the size of our display, increasing the diversity and number of objects presented onscreen, and moving from a button press to eye movement response. Any or all of these changes may have affected the data patterns. Additional experiments that more systematically manipulate response mode versus image diversity might shed light on this issue.

Although future investigations will be necessary to further characterize the relationship between grasp posture and object affordances in determining the nature of visual biases in perihand space, the present study moves this literature forward in important ways. The data from Experiments 2 and 3 suggest that altered vision near the hands occurs with more complex and realistic stimuli than have previously been investigated. In addition, these experiments provide the first evidence that near-hand effects depend not only upon the hands' affordances for grasping, but also on the types of actions objects near the hands afford.

Author Note Data, scripts, and supplementary materials are available through the Open Science Framework, https://osf.io/7yxpg/?view only $=85 \mathrm{f} 85287 \mathrm{e} 04 \mathrm{e} 41229 \mathrm{fl} \mathrm{db} 53769 \mathrm{e} 9 \mathrm{~d} 71 \mathrm{~d}$. This work was supported by a grant from the National Science Foundation (BCS \#1556336) and by an Institutional Development Award from the National Institute of General Medical Sciences of the National Institutes of Health, under grant number P30 GM114748 to L.E.T. We thank Enrique Alvarez Vazquez for technical support throughout these experiments. We also thank our team of undergraduate research assistants: Amran Aden, Emilee Anderson, Steven Blair, Aisha Hassam, Alyx Lundquist, Nicholas Mergen, Kaitlin Morgan, Allison Nadeau, Connor O'Fallon, and Maea Wall. 


\section{Appendix 1}

Table 4 Target images from all experiments

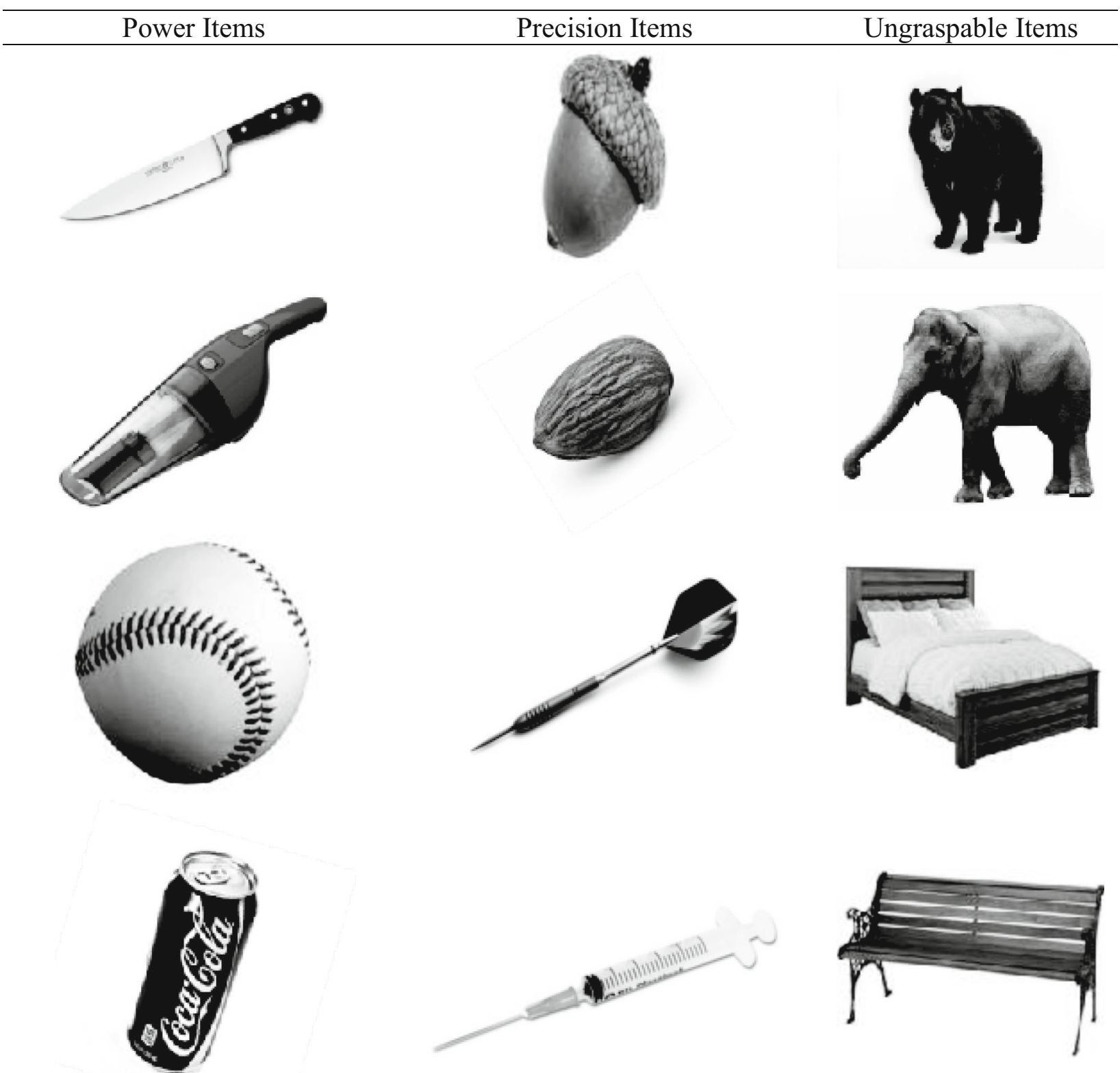


Table 4 (continued)
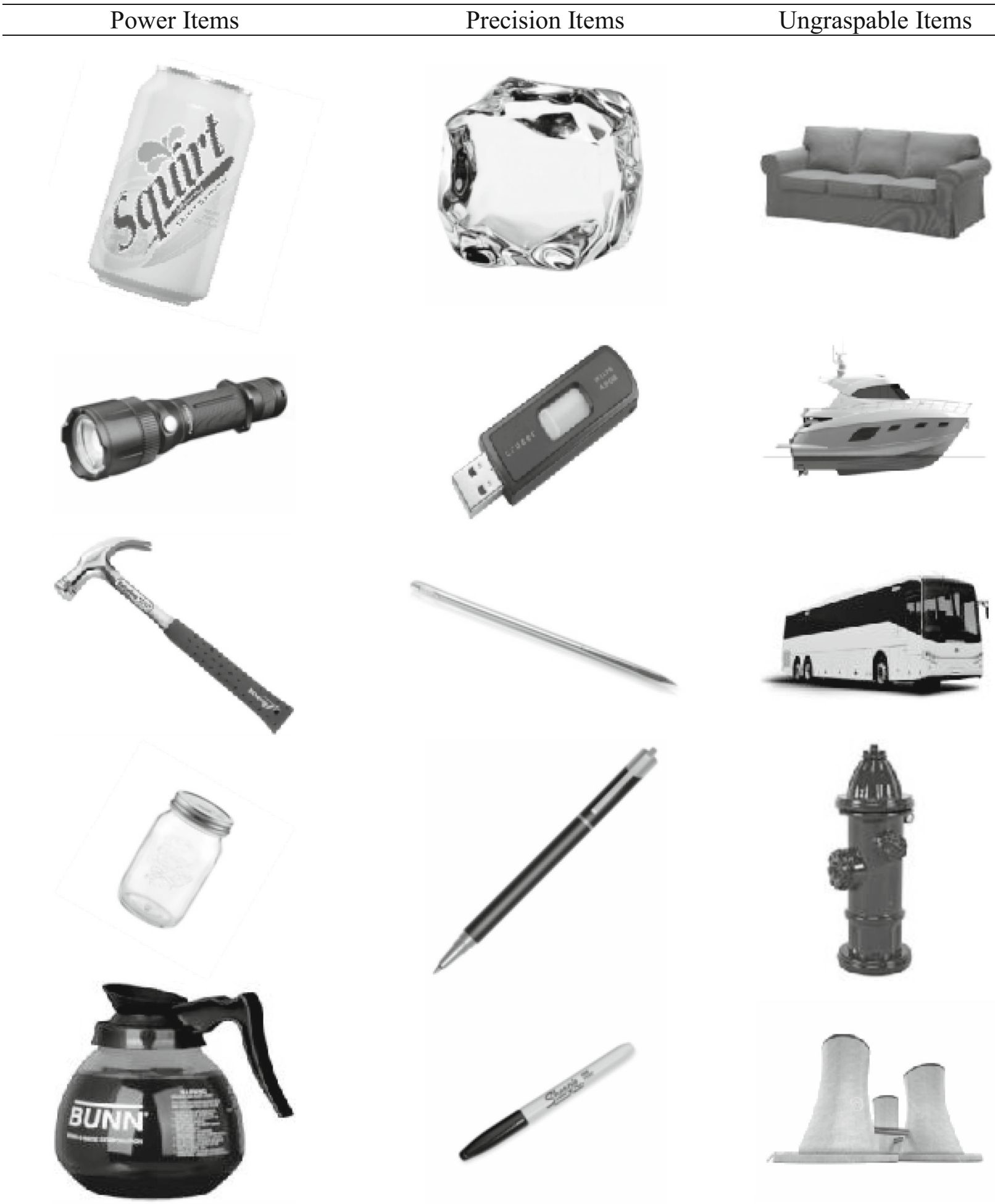
Table 4 (continued)

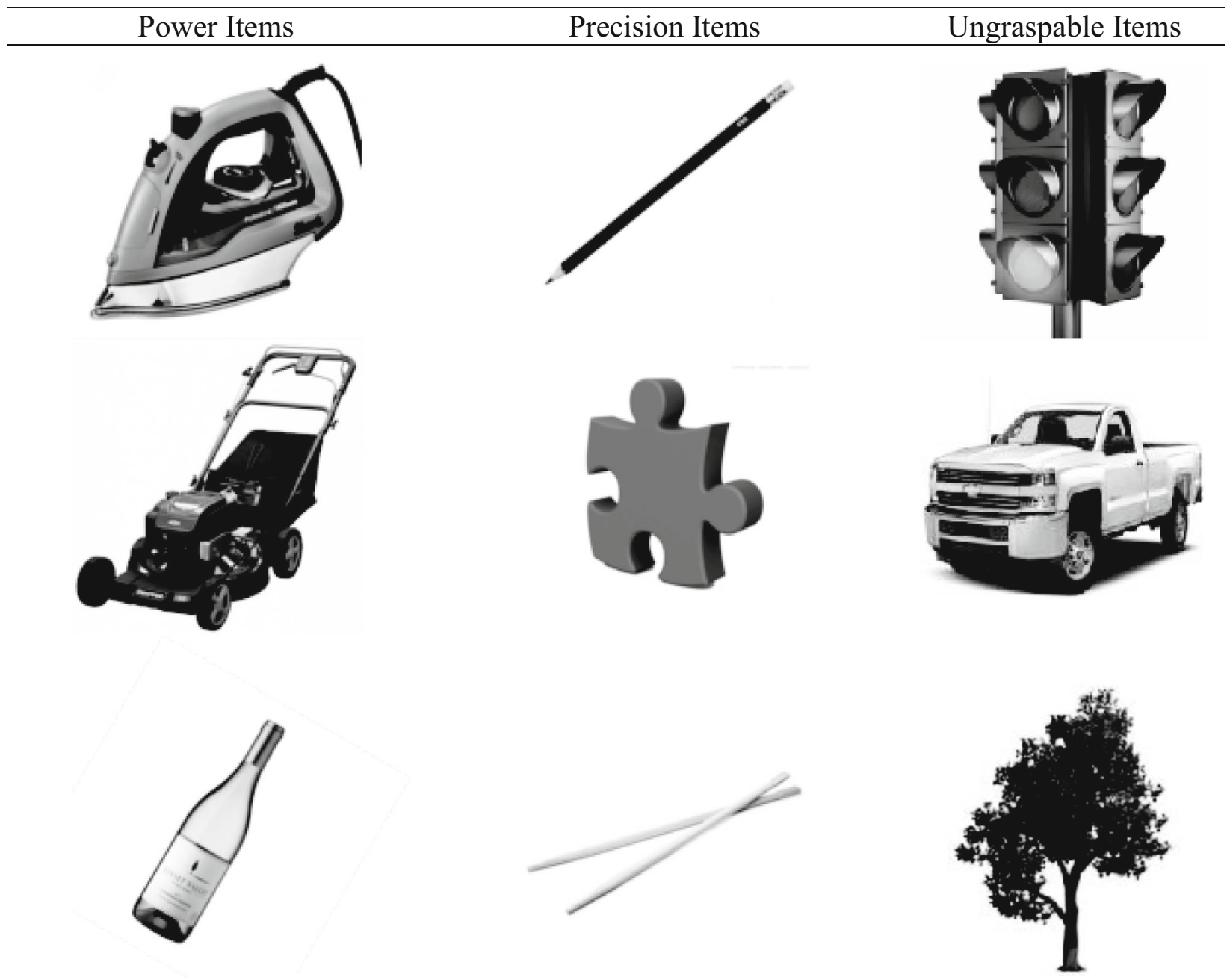




\section{Appendix 2}

Table 5 Experiment 2 ANOVA table with grasp type as a betweensubjects factor

\begin{tabular}{lllll}
\hline Variable & $d f$ & $F$ & $p$ & $\eta_{\mathrm{p}}{ }^{2}$ \\
\hline Hands & 1,78 & 0.04 & .84 & .00 \\
Change Type & 1,78 & 7.50 & .01 & .09 \\
Target Type & 2,156 & 21.40 & $<.001$ & .22 \\
Grasp Type & 1,78 & 0.01 & .93 & .00 \\
Hands $\times$ Change & 1,78 & 6.22 & .02 & .07 \\
Hands $\times$ Target & 2,156 & 6.70 & .002 & .08 \\
Change $\times$ Target & 2,156 & 14.29 & $<.001$ & .15 \\
Hands $\times$ Change $\times$ Target & 2,156 & 1.18 & .31 & .02 \\
Hands $\times$ Grasp Type & 1,78 & 2.46 & .12 & .03 \\
Change $\times$ Grasp Type & 1,78 & 0.38 & .54 & .00 \\
Target $\times$ Grasp Type & 2,156 & 0.24 & .78 & .00 \\
Hands $\times$ Change $\times$ Grasp Type & 1,78 & 0.04 & .85 & .01 \\
Hands $\times$ Target $\times$ Grasp Type & 2,156 & 1.58 & .21 & .02 \\
Change $\times$ Target $\times$ Grasp Type & 2,156 & 1.36 & .26 & .01 \\
Hands $\times$ Change $\times$ Target $\times$ Grasp Type & 2,156 & 0.64 & .53 & .01 \\
\hline
\end{tabular}

\section{References}

Abrams, R. A., Davoli, C. C., Du, F., Knapp, W. H., III, \& Paull, D. (2008). Altered vision near the hands. Cognition, 107, 1035-1047. https://doi.org/10.1016/j.cognition.2007.09.006

Abrams, R. A., \& Weidler, B. J. (2013). Trade-offs in visual processing for stimuli near the hands. Attention, Perception, \& Psychophysics, $76,1242-1252$

Adam, J. J., Bovend'Eerdt, T. J. H., van Dooren, F. E. P., Fischer, M. H., \& Pratt, J. (2012). The closer the better: Hand proximity dynamically affects letter recognition accuracy. Attention, Perception, \& Psychophysics, 74, 1533-1538. https://doi.org/10.3758/s13414012-0339-3

Andringa, R., Boot, W. R., Roque, N. A., \& Ponnaluri, S. (2018) Hand proximity effects are fragile: A useful null result. Cognitive Research: Principles and Implications, 3, 7.

Brockmole, J. R., Davoli, C. C., Abrams, R. A., \& Witt, J. K. (2013). The world within reach: Effects of hand posture and tool use on visual cognition. Current Directions in Psychological Science, 22, 38-44. https://doi.org/10.1177/0963721412465065

Bush, W. S., \& Vecera, S. P. (2014). Differential effect of one versus two hands on visual processing. Cognition, 133, 232-237.

Dosso, J. A., \& Kingstone, A. (2018). The fragility of the near-hand effect. Collabra: Psychology, 4, 27. https://doi.org/10.1525/ collabra. 167

Fagioli, S., Hommel, B., \& Schubotz, R. I. (2007). Intentional control of attention: Action planning primes action-related stimulus dimensions. Psychological Research, 71, 22-29.

Faillenot, I., Decety, J., \& Jeannerod, M. (1999). Perceptual differentiation and category effects in normal object recognition: A PET study. Neuropsychologia, 35, 153-163.
Gerlach, C., Law, I., Gade, A., \& Paulson, O. B. (2000). Categorization and category effects in normal object recognition: A PET Study. Neuropsychologia, 38, 1693-1703.

Goodhew, S. C., \& Clarke, R. (2016). Contributions of parvocellular and magnocellular pathways to visual perception near the hands are not fixed, but can be dynamically altered. Psychonomic Bulletin \& Review, 23, 156-162. https://doi.org/10.3758/s13423-015-0844-1

Goodhew, S. C., Edwards, M., Ferber, S., \& Pratt, J. (2015). Altered visual perception near the hands: A critical review of attentional and neurophysiological models. Neuroscience \& Biobehavioral Reviews, 55, 223-233.

Goodhew, S. C., Fogel, N., \& Pratt, J. (2014). The nature of altered vision near the hands: Evidence for the magnocellular enhancement account from object correspondence through occlusion. Psychonomic Bulletin \& Review, 21, 1452-1458.

Goodhew, S. C., Gozli, D. G., Ferber, S., \& Pratt, J. (2013). Reduced temporal fusion in near-hand space. Psychological Science, 24, 891-900. https://doi.org/10.1177/0956797612463402

Gozli, D. G., West, G. L., \& Pratt, J. (2012). Hand position alters vision by biasing processing through different visual pathways. Cognition, 124, 244-250.

Hommel, B., Müsseler, J., Aschersleben, G., \& Prinz, W. (2001). The Theory of Event Coding (TEC): A framework for perception and action planning. Behavioral and Brain Sciences, 24, 849-878, disc. 878-937. https://doi.org/10.1017/S0140525X01000103

Kelly, S. P., \& Brockmole, J. R. (2014). Hand proximity differentially affects visual working memory for color and orientation in a binding task. Frontiers in Psychology, 5, 318. https://doi.org/10.3389/fpsyg. 2014.00318

Reed, C. L., Betz, R., Garza, J. P., \& Roberts, R. J., Jr. (2010). Grab it! Biased attention in functional hand and tool space. Attention, Perception, \& Psychophysics, 72, 236-245. https://doi.org/10. 3758/APP.72.1.236

Reed, C. L., Grubb, J. D., \& Steele, C. (2006). Hands up: Attentional prioritization of space near the hand. Journal of Experimental Psychology: Human Perception and Performance, 32, 166-177. https://doi.org/10.1037/00961523.32.1.166

SR Research Ltd. (2017). Experiment Builder 2.1.140 [Computer software]. Mississauga, Ontario, Canada: Author.

SR Research Ltd. (2018). EyeLink Data Viewer 3.1.246 [Computer software]. Mississauga, Ontario, Canada: Author.

Sun, H.-M., \& Thomas, L. E. (2013). Biased attention near another's hand following joint action. Frontiers in Psychology, 4, 443. https://doi.org/10.3389/fpsyg.2013.00443

Symes, E., Tucker, M., Ellis, R., Vainio, L., \& Ottoboni, G. (2008). Grasp preparation improves change detection for congruent objects. Journal of Experimental Psychology: Human Perception and Performance, 34, 854-871. https://doi.org/10.1037/0096-1523.34. 4.854

Taylor, J. E. T., Gozli, D. G., Chan, D., Huffman, G., \& Pratt, J. (2015). A touchy subject: Advancing the modulated visual pathways account of altered vision near the hand. Translational Neuroscience, 6, 1-7.

Thomas, L. E. (2013). Grasp posture modulates attentional prioritization of space near the hands. Frontiers in Psychology, 4, 312.

Thomas, L. E. (2015). Grasp posture alters visual processing biases near the hands. Psychological Science, 26, 625-632.

Thomas, L. E. (2017). Action experience drives visual-processing biases near the hands. Psychological Science, 28, 124-131.

Tseng, P., Bridgeman, B., \& Juan, C. H. (2012). Take the matter into your own hands: A brief review of the effect of nearby hands on visual processing. Vision Research, 72, 74-77.

Tucker, M., \& Ellis, R. (1998). On the relations between seen objects and components of potential actions. Journal of Experimental 
Psychology: Human Perception and Performance, 24, 830-846. https://doi.org/10.1037/0096-1523.24.3.830

Tucker, M., \& Ellis, R. (2004). Action priming by briefly presented objects. Acta Psychologica, 116, 185-203.

Wykowska, A., Schubö, A., \& Hommel, B. (2009). How you move is what you see: Action planning biases selection in visual search.
Journal of Experimental Psychology: Human Perception and Performance, 35, 1755-1769. https://doi.org/10.1037/a0016798

Publisher's note Springer Nature remains neutral with regard to jurisdictional claims in published maps and institutional affiliations. 